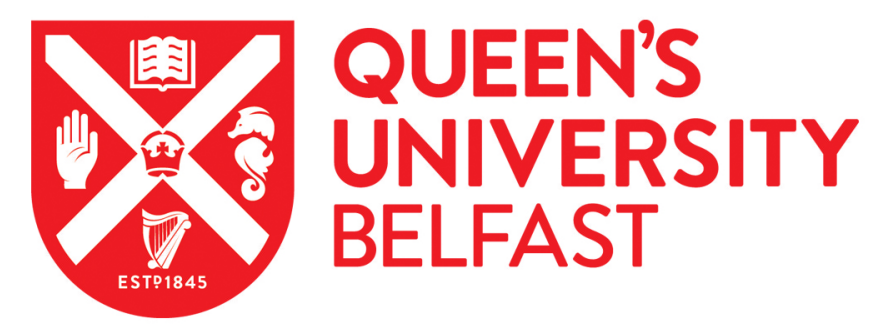

\title{
Influence of azithromycin and allograft rejection on the post-lung transplant microbiota
}

Spence, C. D., Vanaudenaerde, B., Einarsson, G. G., Mcdonough, J., Lee, A. J., Johnston, E., Verleden, G. M., Elborn, J. S., Dupont, L. J., Van Herck, A., Gilpin, D. F., Vos, R., Tunney, M. M., \& Verleden, S. E. (2019). Influence of azithromycin and allograft rejection on the post-lung transplant microbiota. Journal of Heart and Lung Transplantation, 39(2), 176. https://doi.org/10.1016/j.healun.2019.11.007

Published in:

Journal of Heart and Lung Transplantation

Document Version:

Peer reviewed version

Queen's University Belfast - Research Portal:

Link to publication record in Queen's University Belfast Research Portal

Publisher rights

Copyright 2019 Elsevier

This manuscript is distributed under a Creative Commons Attribution-NonCommercial-NoDerivs License

(https://creativecommons.org/licenses/by-nc-nd/4.0/), which permits distribution and reproduction for non-commercial purposes, provided the author and source are cited.

\section{General rights}

Copyright for the publications made accessible via the Queen's University Belfast Research Portal is retained by the author(s) and / or other copyright owners and it is a condition of accessing these publications that users recognise and abide by the legal requirements associated with these rights.

Take down policy

The Research Portal is Queen's institutional repository that provides access to Queen's research output. Every effort has been made to ensure that content in the Research Portal does not infringe any person's rights, or applicable UK laws. If you discover content in the Research Portal that you believe breaches copyright or violates any law, please contact openaccess@qub.ac.uk. 
Influence of azithromycin and allograft rejection on the post-lung transplant microbiota

Christopher D Spence ${ }^{1 *}$, Bart Vanaudenaerde ${ }^{2 *}$, Gísli G Einarsson³ ${ }^{3}$ John Mcdonough², Andrew J Lee ${ }^{1}$, Elinor Johnston ${ }^{1}$, Geert M Verleden ${ }^{2}$, J Stuart Elborn ${ }^{3}$, Lieven J Dupont ${ }^{2}$, Anke Van Herck ${ }^{2}$, Deirdre F Gilpin ${ }^{1}$, Robin Vos ${ }^{2}$, Michael M Tunney ${ }^{1 *}$, Stijn E Verleden ${ }^{2 *}$

${ }^{1}$ School of Pharmacy, Queen's University Belfast, Belfast, UK.

${ }^{2}$ Leuven Lung Transplant Unit, Department of Chronic Diseases, Metabolism and Ageing, KU Leuven, Leuven, Belgium

${ }^{3}$ School of Medicine, Dentistry and Biomedical Sciences, Queen's University Belfast, Belfast, UK.

*Authors contributed equally

Word count: 2936

Address for correspondence:

Dr Stijn Verleden

$\mathrm{K} U$ Leuven

Lung Transplantation Unit, O\&N1, BOX 706

49 Herestraat, B-3000 Leuven, Belgium

Tel: + 3216377459 Fax: + 3216330806

E-mail: stijn.verleden@kuleuven.be

This article has an online data supplement 


\section{ABSTRACT}

Background: Alterations in the lung microbiota may drive disease development and progression in patients with chronic respiratory diseases. Following lung transplantation (LTx), azithromycin is used to both treat and prevent chronic lung allograft dysfunction (CLAD). The objective of this study was to determine the association between azithromycin use, CLAD, acute rejection, airway inflammation and bacterial microbiota composition and structure after LTX.

Methods: Bronchoalveolar lavage (BAL) samples ( $n=219)$ from 69 LTx recipients (azithromycin, $\mathrm{n}=32$; placebo, $\mathrm{n}=37$ ) from a previously conducted randomized placebocontrolled trial with azithromycin were analysed. Samples were collected at discharge, 1 and 2 years following randomization and at CLAD diagnosis. Bacterial microbial community composition and structure was determined using 16S rRNA gene sequencing and associated with clinically important variables.

Results: At discharge and following 1 and 2 years of azithromycin therapy, no clear differences in microbial community composition or overall diversity were observed. Moreover, no changes in microbiota composition were observed in CLAD phenotypes. However, acute rejection was associated with a reduction in community diversity $(p=0.0009)$. Significant correlations were observed between microbiota composition, overall diversity and levels of inflammatory cytokines in BAL, particularly CXCL8.

Conclusions: Chronic azithromycin usage did not disturb the bacterial microbiota. However, acute rejection episodes were associated with bacterial dysbiosis.

Word count: 206 words 


\section{INTRODUCTION}

Due to its direct contact with the external environment, the lung is uniquely susceptible to microbial invasion. Unsurprisingly, diverse polymicrobial communities have been observed in the airways of both healthy subjects and those with chronic respiratory diseases, including cystic fibrosis (CF), chronic obstructive pulmonary disease (COPD) and idiopathic pulmonary fibrosis (IPF) (1-3). Moreover, there is a dynamic interaction between the airway microbiota and immune cell activation, with characteristics of specific lung microbiota aligning with distinct immune cell gene expression profiles, as shown in COPD and IPF (2,3). A number of non-interventional studies have reported that post-LTx bacterial density is higher and may have altered diversity compared to the pre-LTx microbiota (4-7). Moreover, disturbance of the microbiota may be associated with poor post-transplant outcome.

Azithromycin, a neo-macrolide antibiotic, is increasingly used in the management of chronic respiratory diseases such as COPD, CF, non-CF bronchiectasis, diffuse panbronchiolitis and asthma to reduce exacerbation frequency and increase quality of life for patients $(9,10)$. The effects of azithromycin are diverse and not fully understood but are known to include antibiotic, anti-inflammatory and immunomodulatory components. These beneficial effect of azithromycin may also be related to modulation of the microbiota or due to specific interactions with Pseudomonas, including reducing biofilm formation or inhibition of bacterial quorum-sensing (11-12).

Given the need for daily immunosuppression, LTx recipients could be particularly susceptible to changes in the lung microbiota. Colonization of the lung allograft with microorganisms such as $P$. aeruginosa and $A$. fumigatus is common and increases the risk of subsequent chronic lung allograft dysfunction (CLAD) and mortality $(13,14)$. Azithromycin is currently first line treatment when CLAD is suspected (15) and two randomized controlled 
trials have demonstrated its effect in improving pulmonary function and prolonging CLADfree and graft survival $(16,17)$. We used bronchoalveolar lavage (BAL) samples collected during a previously conducted RCT with azithromycin to determine the effects of azithromycin on the post- LTx microbiota. More specifically, we assessed if the previously reported beneficial effect of azithromycin in prolonging CLAD-free and graft survival could be explained by a reduction in the bacterial load or the reduced presence of certain microorganisms (i.e. Pseudomonas). We also determined if there was an association between microbiota composition and clinically important variables, such as acute rejection, CLAD (phenotypes) and airway inflammation.

\section{MATERIAL \& METHODS}

\section{Study population}

This work is a post-hoc analysis of samples collected during a randomised, double-blind, placebo-controlled trial of oral azithromycin to prevent CLAD, conducted in UZ Gasthuisberg, Leuven, Belgium (16). In brief (figure 1), 40 patients were randomly assigned to the treatment arm (azithromycin) and 43 to the placebo arm of the study. Patients assigned to the treatment arm received azithromycin $250 \mathrm{mg}$ (Pfizer, Sandwich, UK) thrice weekly from discharge posttransplant. CLAD was diagnosed as a persistent (>3 weeks) forced expiratory volume in 1 second $\left(F E V_{1}\right)$ decline $\geq 20 \%$ of the two best post-operative values in the absence of identifiable causes. Further post-hoc subdivision for the two main CLAD phenotypes, restrictive allograft syndrome (RAS) and bronchiolitis obliterans syndrome (BOS), were made based on spirometry and radiological findings (online supplement), according to the new 
consensus guidelines (18). When CLAD was suspected, the study drug was stopped (without unblinding) and the patient was initiated on open-label treatment with azithromycin. Samples from the placebo group taken after the date of switch were not included in the comparison between azithromycin and placebo. The initial azithromycin study was approved by the hospital's ethical committee (ML3199), with this post-hoc analysis approved separately (S61168). Censoring of patient follow-up was completed on the $1^{\text {st }}$ January 2017.

\section{Collection and processing of BAL samples}

Samples were obtained during surveillance bronchoscopy with BAL at specific time-points after transplantation (discharge, 1 year, 2 years post-transplant) and for clinical indications in cases of suspected CLAD diagnosis. Discharge samples were used as baseline as none of the patients were treated with azithromycin at that time. Patients who had an infection at the given timepoint of BAL (elevated CRP, new positive culture or positive PCR and chest X-ray alterations), were a priori excluded from this microbiota analysis with samples not sequenced. BAL was performed in a subsegmental bronchus of the right middle lobe or lingula with two $50 \mathrm{~mL}$ aliquots of saline, which were pooled for routine total and cell differential counts and microbiology. Transbronchial biopsies were taken at discharge or in case of clinical symptoms (dyspnoea, fever, loss in $\mathrm{FEV}_{1}$ ) and was scored for acute rejection (A0-A4) and lymphocytic bronchiolitis (B0-B2R) according to ISHLT guidelines by an experienced LTX pathologist (19). BAL was centrifuged $\left(1500 \mathrm{rpm}, 4^{\circ} \mathrm{C}, 10\right.$ minutes), with supernatant and cell pellet stored separately. The pellet was suspended in $500 \mu \mathrm{l}$ trizol and stored at $-80^{\circ} \mathrm{C}$. Supernatants were used to measure interleukin-6 (IL-6) and C-X-C Motif Chemokine Ligand 8 (CXCL-8) in duplicate via ELISA according to the manufacturers' instructions (Life Sciences). 
After further centrifugation at approximately $10000 \mathrm{rpm}$ for 5 minutes, the resulting pellet plus $300 \mu \mathrm{L}$ of accompanying fluid was processed for pre-lysis and DNA extraction using the MagNA pure 96 (Roche Diagnostics Ltd., Burgess Hill, UK) automated DNA extraction platform, using a DNA and viral NA small volume kit (Roche Diagnostics Ltd., Burgess Hill, UK). Samples were stored at $-20^{\circ} \mathrm{C}$ prior to quantitative $\mathrm{PCR}$ (qPCR) and sequencing.

\section{Quantitative PCR and Illumina MiSeq Sequencing}

Total bacterial load in BAL DNA extracts was determined in triplicate together with positive, negative and non-template controls via quantitative PCR (qPCR), using a well-established primer and probe set targeting the bacterial 16S rRNA gene (20). Reactions were performed in $10 \mu \mathrm{L}$ volumes on the LightCycler 480 II platform (Roche Diagnostics Ltd., Burgess Hill, UK). The number of $16 \mathrm{~S}$ rRNA gene copies per $\mathrm{mL}$ of BAL were calculated using the absolute quantification analysis program using a standard curve prepared from purified $16 \mathrm{~S}$ amplicons, and is referred to as bacterial burden.

Paired-end Illumina sequencing of the bacterial 16S rRNA gene from BAL DNA extracts was carried out using the 515F/806R primer set (21), targeting the V4 hypervariable region. Library preparation was carried out using an optimised version of a previously published fourstep PCR protocol (22). Following purification and confirmation of amplification via gel electrophoresis, amplicons were quantified and pooled at 20nM. Following gel purification, sequencing was performed on the Illumina MiSeq platform using 500-cycle V2 reagent kits (Illumina UK, Cambridge, UK).

Paired-end 251 BP reads were generated using the GenerateFASTQ workflow and the FASTQ-only analysis format. Downstream processing of sequences was carried out using 
QIIME (23). Sequencing was carried out alongside positive and negative controls to monitor inter-run variability and monitor for potential reagent / user-introduced contaminants.

More details are provided in the supplementary material. Sequence data is reported in the SRA database (accession number SRP158593).

\section{Statistical analysis}

Normality was assessed via D'Agostino and Pearson test. Unpaired analysis (azithromycin vs placebo, CLAD status) was performed using Mann-Whitney $\mathrm{U}$ testing. Longitudinal changes in microbiota composition, community metrics and $16 \mathrm{~S}$ copy numbers within treatment groups were analyzed using the Kruskal-Wallis ANOVA, with Dunn's multiple comparison test posthoc. A mixed effects model was used to confirm the association between acute rejection and Shannon diversity, with subject as random effect. Correlations were performed via Spearman's rank test with false discovery rate correction for multiple testing. All tests were performed using GraphPad Prism, (La Jolla, California, USA) or SAS 9.4. A p-value $<0.05$ is considered statistically significant.

\section{RESULTS}

A total of 219 samples from 70 patients (table 1) were processed for microbiota analysis (figure 1). Prior to quality filtering and removal of suspected contaminants from samples, $5,383,750$ sequences were generated, representing 39,859 unique OTUs. After filtering, a mean number of 24,526 (s.d. 25,530 ; range $8-178,396$ ) sequences per sample were retained. Ten positive and negative controls were included, generating mean numbers of 59, 205 and 
2,819 sequences per control, respectively. Full details are provided in the supplemental material.

Results could not be generated for 22 samples, due to failure of library preparation PCR or where sequences detected were removed as background / contaminant taxa following filtering of sequences or normalization to a depth of 2,000 sequences for downstream analysis. Therefore, a total of 197 samples from 69 patients were included in the analysis. Of these samples, 97 were from the azithromycin arm ( $n=32$ patients) and 100 from the placebo arm ( $n=37$ patients). The most abundant genera across all samples was Pseudomonas (mean relative abundance, [RA] 30.3\%; 86\% of samples), followed by Streptococcus (mean RA 20.1\%; $93 \%$ of samples). See figure E1 of the online supplement for a visual representation of incidence and prevalence of each genera.

\section{Impact of azithromycin on the post-LTx microbiota}

CLAD-free survival tended to be longer in patients in the azithromycin vs placebo group ( $p=0.069$, median CLAD free survival of 7.71 vs 4.98 years post-transplant). However, there was no difference in five year survival ( 84 vs $78 \%, p=0.25$ ). At discharge, there were no significant differences observed between the azithromycin and placebo cohorts in either total bacterial burden measured by qPCR, Shannon diversity / evenness and RA of any specific genera present at a mean abundance $\geq 1 \%$. Although bacterial burden increased in both placebo $(p=0.020)$ and tended to increase in azithromycin-treated $(p=0.099)$ patients at 1 year post-transplant, there were no differences in Shannon diversity / evenness between cohorts. Statistically significant differences in relative abundances were observed between both groups for a number of low abundant genera, including unclassified Enterobacteriaceae $(p=0.042)$, Neisseria $(p=0.0072)$ and Enterococcus $(p=0.042)$. Two years after randomization, 
bacterial burden, Shannon diversity and evenness were similar in the azithromycin and placebo group. However, there was a decrease in RA of Pseudomonas $(p=0.049)$, accompanied by an increase in RA of Streptococcus $(p=0.045)$, Lactobacillales $(p=0.032)$ and Gemellaceae $(p=0.036)$ in the azithromycin group (figure 2$)$. However, these associations were not robust when correcting for multiple testing (all $p>0.05$ ). In patients that were switched from placebo to open label azithromycin $(n=19)$, there was no difference in bacterial burden, Shannon diversity / evenness or RA of specific genera in samples pre- and postswitching (median time between switch samples: 209 days; IQR: 109-383, figure E2 online supplement).

\section{Relationship between post-LTx microbiota and rejection}

When assessing changes in the microbiota across different time points, independent of azithromycin use, there was an increase in RA of Actinomyces $(p=0.001)$, Prevotella $(p<0.001)$ and Veillonella $(p<0.001)$ and a trend towards a decrease in Pseudomonas RA $(p=0.081)$ between samples at discharge and 1 year following transplant. No additional differences in RA, diversity or eveness between 1 and 2 years post-transplant were observed. In contrast, a significant decrease in community richness was detected between discharge and 1 year $(p=0.04)$, in addition to increased total bacterial burden $(p=0.01)$. Both of these measurements did not change further between 1 and 2 years post-transplant (figure 3 ).

For 86 of the collected BAL samples, a transbronchial biopsy was also collected, 26 (29\%) of which demonstrated acute cellular rejection $(A 1, n=12 ; A \geq 2, n=14)$. There was no difference in bacterial burden ( $p=0.59)$ between patients based on acute rejection status; however, Shannon diversity $(p=0.002)$, evenness $(p=0.0034)$ and richness $(p=0.02)$ were 
significantly lower in patients with acute rejection compared to patients free from acute rejection (figure 4), which could not be attributed to a difference in relative abundance of any specific genera. In contrast, there was no association between lymphocytic bronchiolitis (94 biopsies included of which 15 demonstrated lymphocytic bronchiolitis) and Shannon diversity $(p=0.27)$ and evenness $(p=0.34)$. The observed association between the post-LTx microbiota and acute rejection proved to be independent of confounding variables in adjusted analysis, taking azithromycin treatment and presence of lymphocytic bronchiolitis on transbronchial biopsy into account $(p=0.0009)$.

Within this study, 24 patients contributed a BAL sample at or near CLAD diagnosis $( \pm 3$ months) of which 17 (71\%) were already supplemented with azithromycin before CLAD diagnosis. There were no differences in Shannon diversity $(p=0.52)$ or evenness $(p=0.34)$ when samples at baseline, 1 year, 2 years and at CLAD diagnosis were compared. To assess whether microbial diversity influenced subsequent CLAD development, BAL microbiota at discharge, 1 year and 2 years was compared in patients who developed CLAD during follow up (even beyond the 2 year treatment period) versus patients who never developed CLAD. No differences were observed between bacterial burden, richness, evenness, overall diversity or specific genera at any timepoint (all $p>0.05$ ). Furthermore, when subdividing CLAD into BOS $(n=16)$ or RAS $(n=8)$ phenotype, there were no differences observed between phenotypes (all $p>0.05$ ) (figure $3 A$ ).

\section{Relationship between post-LTx microbiota and airway inflammation}

Unadjusted analysis demonstrated significant correlations between taxa with a mean RA $>1 \%$ and total and differential cell counts, BAL IL-6, BAL CXCL-8, systemic c-reactive protein (CRP) 
and $\mathrm{FEV}_{1}$ in the entire population (either treated or untreated) (figure 5). Adjusting for multiple testing, demonstrated that only CXCL-8 levels were positively correlated with RA of Pseudomonas $(p=0.008)$, and negatively correlated with RA of Streptococcus $(p=0.002)$, Actinomyces $(p<0.0001)$, Prevotella $(p<0.0001)$, Veillonella $(p<0.0001)$, and also with unclassified Gemellaceae $(p=0.009)$ and Lactobacillales $(p=0.026)$. Furthermore, Shannon evenness $(p<0.0001)$ and diversity $(p=0.003)$ showed significant inverse correlations with BAL CXCL-8. After adjustment, there was also a negative correlation between IL- 6 and Actinomyces RA ( $p=0.019)$, but not with any other microbiota parameters.

\section{DISCUSSION}

This post-hoc analysis of the bacterial microbiota in BAL samples from a prior randomized placebo-controlled trial of azithromycin for prevention of CLAD, demonstrates that azithromycin did not impact overall diversity or relative abundance of specific bacterial genera. Furthermore, acute rejection, but not CLAD was associated with a significant loss in microbial diversity. Finally, airway CXCL8 expression strongly correlated with multiple features of microbiota composition and overall diversity.

Azithromycin has become an important component of post-LTx management, with multiple studies reporting its beneficial effects, supported by 2 randomized placebocontrolled trials $(16,17)$. In addition to its traditional antibacterial affects, azithromycin is now known to exhibit anti-inflammatory and immunomodulatory effects, as well as specific anti-pseudomonal effects, including decreasing biofilm formation and inhibition of bacterial quorum sensing (11-12). In the present study, azithromycin did not impact either overall microbiota diversity or relative abundance of specific bacterial genera, despite its positive effects on prolonging CLAD-free and overall graft survival $(16,17)$. This contrasts with findings 
in other respiratory conditions, including asthma and COPD, where azithromycin use resulted in lower microbial diversity (24-25). However, both these studies used more frequent daily dosing. Furthermore, these studies followed patients for shorter periods than the current work (6-8 weeks); these findings could represent an initial short-term change in the microbiota, that reverts to pre-treatment composition after longer-term treatment, as has been shown in other conditions following treatment cessation (6). This loss of diversity has been shown to correlate with negative patient outcomes in conditions such as CF (1), COPD (2) and IPF (3) and so it is likely a positive finding that the dose used in the current study ( $250 \mathrm{mg}$ thrice weekly) is sufficient to improve long-term patient outcomes without affecting microbial diversity. Therefore, it seems likely that anti-inflammatory and not antimicrobial effects are responsible for the beneficial effects of azithromycin in lung transplant patients, as demonstrated by a decreased pro-inflammatory environment (i.e. IL-17, CXCL-8, neutrophil count) following 3 months treatment with azithromycin $(16,26)$. However, it is also possible that the effects of azithromycin in a severely immunocompromised population such as LTx patients is different to that in a COPD or CF population.

There are limited publications with respect to what changes occur in the microbiota at the onset of CLAD. Willner and colleagues reported a reduction in diversity with development of BOS (6). Results from a further study demonstrated that an actinobacteriadominated microbiota, enriched with gram positive organisms, inferred a reduced risk of acute rejection and $\mathrm{BOS}$, compared with a microbiota dominated by proteobacteria or a mixed microbiota (27). In the present work, no changes in overall diversity or bacterial microbiota composition were associated with CLAD onset. Possible explanations include the design of the study where our initial focus was the influence of azithromycin on the posttransplant microbiome, the possible heterogeneity in CLAD phenotypes and the lack of 
statistical power for the CLAD analysis. Although the technology used is the current golden standard, given the continuous technological advancements, it seems impossible to exclude that future technology will be able to demonstrate more subtle differences

However, acute rejection was associated with reduced community evenness and diversity, without clear changes at genus level. This suggests that loss of diversity may be due to changes in the unique microbiota of each patient. This relationship between acute rejection and microbial (gut) dysbiosis has already been demonstrated following vessel and liver transplantation $(28,29)$.

We also determined a possible link between microbiota composition and post-LTx BAL inflammation. Pseudomonas RA correlated with increased CXCL-8 levels, while RA of other genera, e.g. Prevotella, Streptococcus, Veillonella and Actinomyces correlated with reduced CXCL-8 levels in BAL. This supports recently published work (29) illustrating that catabolic remodelling of the lung after LTx is associated with a microbiota dominated by proinflammatory bacteria (e.g. Staphylococcus and Pseudomonas) which were linked to high expression of metallopeptidases 7, 9 and 12 . Conversely, anabolic remodelling was linked to bacteria such as Prevotella, Streptococcus, and Veillonella. Moreover, a robust association with myeloid derived suppressor cells and the BAL microbiota has also been demonstrated (9). Our study further supports the association between changes in microbiota composition and airway inflammation, with an especially strong association with CXCL-8, one of the main neutrophil chemo-attractants.

There are a number of limitations to this study. Firstly, we did not have a group of healthy non-LTx patients; therefore, previously identified changes in the microbiota between LTx and non-LTx patients could not be investigated. Secondly, we did not have bronchoscope washes from the time of sampling and cannot rule out the possibility of bronchoscope contamination. 
Unfortunately, since this study was designed retrospectively after the original RCT was carried out, it was not possible to obtain these controls. However, we stringently checked for any potential contamination and robust controls were employed in all downstream processing of BAL samples. We opted to use samples collected during a previous adequately powered RCT to avoid changes in clinical practice and obtain a fair comparison between both cohorts. As a result, for some of the analyses performed, power was limited. We also opted to study the long-term rather than short-term effect (3-6 months) as we were primarily interested in determining if long-term use of azithromycin resulted in a change in bacterial microbiota composition. We acknowledge that short-term changes could also be of interest. A further potential confounder is the use of antibiotics and anti-rejection therapy; however, samples were taken before any potential therapy was initiated per design of the study. Moreover, in the 37 patients who received antibiotics during the 2 year randomisation period $(n=76$ events), the median time between last antibiotic use and collection of the sample analysed in this study was 177 days (57-274 days), decreasing the potential of a confounding effect.

In conclusion, this work has shown that long-term azithromycin treatment did not impact overall bacterial community diversity. Acute rejection but not CLAD was associated with reduced BAL bacterial diversity. Finally, bacterial microbiota composition and structure correlated with inflammation, particularly CXCL-8 which could contribute to allograft remodelling or rejection.

\section{Acknowledgement}

The authors would like to thank the members of the lab of pneumology for collection of the samples. 


\section{Disclosures}

None of the authors have anything to disclose according to the regulation of the Journal of Heart and Lung Transplantation.

\section{Funding}

Sources of support: C.D.S. supported by a Department for Employment and Learning (Northern Ireland) studentship. M.M.T. and J.S.E are supported by the Health and Social Care Northern Ireland (HSC-NI) Research and Development office (COM4978/14). SEV, GMV and BMV are supported by FWO (12G8718N and G083818N).SEV, BMV and GMV are also supported by KU Leuven (C24/18/073 and C24/050). GMV is supported by the Broere foundation.

\section{REFERENCES}

1. Einarsson GG, Comer DM, Mcllreavey L, et al. Community dynamics and the lower airway microbiota in stable chronic obstructive pulmonary disease, smokers and healthy non-smokers. Thorax 2016; 71: 795-803.

2. Sze MA, Dimitriu PA, Suzuki M, et al. Host response to the lung microbiome in chronic obstructive pulmonary disease. Am J Respir Crit Care Med 2015; 192: 438-445.

3. Molyneaux PL, Willis-Owen SAG, Cox MJ, et al. Host-microbial interactions in idiopathic pulmonary fibrosis. Am J Respir Crit Care Med 2017; 195: 1640-1650.

4. Sharma NS, Wille KM, Athira S, et al. Distal airway microbiome is associated with immunoregulatory myeloid cell responses in lung transplant recipients. J Heart Lung Transplant 2018; 37: 206-216. 
5. Charlson ES, Diamond JM, Bittinger K, et al. Lung-enriched organisms and aberrant bacterial and fungal respiratory microbiota after lung transplant. Am J Respir Crit Care Med 2012; 186: 536-545.

6. Willner DL, Hugenholtz P, Yerkovich St et al. Reestablishment of recipient-associated microbiota in the lung allograft is linked to reduced risk of bronchiolitis obliterans syndrome. Am J Respir Crit Care Med 2013; 187: 640-647.

7. Borewicz K, Pragman AA, Kim HB, et al. Longitudinal analysis of the lung microbiome in lung transplantation. FEMS Microbiol Lett 2013; 339: 57-65.

8. Gibson PG, Yang IA, Upham JW, et al. Effect of azithromycin on asthma exacerbations and quality of life in adults with persistent uncontrolled asthma (AMAZES): a randomised, double-blind, placebo-controlled trial. Lancet 2017; 390: 659-668.

9. Albert RK, Connett J, Bailey WC, et al. Azithromycin for prevention of exacerbations of COPD. N Engl J Med 2011; 365: 689-698.

10. Valery PC, Morris PS, Byrnes CA, et al. Long-term azithromycin for Indigenous children with non-cystic-fibrosis bronchiectasis or chronic suppurative lung disease (Bronchiectasis Intervention Study): a multicentre, double-blind, randomised controlled trial. Lancet Respir Med. 2013; 1: 610-620.

11. Vos R, Vanaudenaerde BM, Verleden SE, et al. Anti-inflammatory and immunomodulatory properties of azithromycin involved in treatment and prevention of chronic lung allograft rejection. Transplantation 2012; 94: 101-109.

12. Parnham MJ, Haber VE, Giamarellos-Bourboulis EJ, et al. Azithromycin: Mechanisms of action and their relevance for clinical applications. Pharmacol Ther 2014; 143: $225-$ 245. 
13. Vos R, Vanaudenaerde BM, Geudens N, et al. Pseudomonal airway colonisation: risk factor for bronchiolitis obliterans syndrome after lung transplantation? Eur Respir J 2008; 31: 1037-1045.

14. Gregson AL, Wang XY, Weigt SS, et al. Interaction between Pseudomonas and CXC chemokines increases risk of bronchiolitis obliterans syndrome and death in lung transplantation. Am J Respir Crit Care Med 2013; 187: 518-526.

15. Meyer KC, Raghu G, Verleden GM, et al. An international ISHLT/ATS/ERS clinical practice guideline: diagnosis and management of bronchiolitis obliterans syndrome. Eur Respir J 2014; 44: 1479-1503.

16. Vos R, Vanaudenaerde BM, Verleden $\mathrm{SE}$, et al. A randomised controlled trial of azithromycin to prevent chronic rejection after lung transplantation. Eur Respir J 2011; 37: 164-172.

17. Corris PA, Ryan VA, Small T, et al. A randomised controlled trial of azithromycin therapy in bronchiolitis obliterans syndrome (BOS) post lung transplantation. Thorax 2015; 70: 442-U150.

18. Verleden GM, Glanville AR, Lease ED, et al. Chronic lung allograft dysfunction: Definition, diagnostic criteria, and approaches to treatment-A consensus report from the Pulmonary Council of the ISHLT. J Heart Lung Transplant. 2019

19. Stewart S, Fishbein MC, Snell GI, et al. Revision of the 1996 working formulation for the standardization of nomenclature in the diagnosis of lung rejection. Journal of Heart and Lung Transplantation 2007; 26: 1229-1242.

20. Nadkarni MA, Martin FE, Jacques NA, et al. Determination of bacterial load by realtime PCR using a broad-range (universal) probe and primers set. Microbiology. 2002; 148: $257-266$. 
21. Caporaso JG, Lauber CL, Walters WA, et al. Ultra-high-throughput microbial community analysis on the Illumina HiSeq and MiSeq platforms. Isme J 2012; 6: 16211624

22. Lundberg DS, Yourstone S, Mieczkowski $P$, et al. Practical innovations for highthroughput amplicon sequencing. Nat Methods 2013; 10: 999-1002.

23. Caporaso JG, Kuczynski J, Stombaugh J, et al. QIIME allows analysis of high-throughput community sequencing data. Nat Methods 2010; 7: 335-336.

24. Slater M, Rivett DW, Williams L, et al. The impact of azithromycin therapy on the airway microbiota in asthma. Thorax 2014; 69: 673-674.

25. Segal LN, Clemente JC, Wu BG, et al. Randomised, double-blind, placebo-controlled trial with azithromycin selects for anti-inflammatory microbial metabolites in the emphysematous lung. Thorax 2017; 72: 13-22.

26. Vos R, Verleden SE, Ruttens D, et al. Azithromycin and the treatment of lymphocytic airway inflammation after lung transplantation. Am J Transplant. 2014 Dec;14(12):2736-48.

27. Schott C, Weigt SS, Turturice BA, et al. Bronchiolitis obliterans syndrome susceptibility and the pulmonary microbiome. J Heart Lung Transplant 2018;37(9):1131-1140.

28. Rey K, Manku S, Enns W, et al. Disruption of the gut microbiota with antibiotics exacerbates acute vascular rejection. Transplantation 2018; 102: 1085-1095.

29. Ren ZG, Jiang JW, Lu HF, et al. Intestinal microbial variation may predict early acute rejection after liver transplantation in rats. Transplantation 2014; 98: 844-852.

30. Mouraux S, Bernasconi E, Pattaroni C, et al. Airway microbiota signals anabolic and catabolic remodeling in the transplanted lung. J Allergy Clin Immunol 2018; 141: 718729. 
Table 1: patient characteristics

Age at transplant, $Y$

Underlying disease, $\mathbf{N}(\%)$

Emphysema $+\alpha 1$ ATD

Pulmonary fibrosis

$\mathrm{CF}+\mathrm{BRECT}$

Pulmonary hypertension

Other

Type of transplant, $\mathbf{N}(\%)$

HLTX

SLTX

SSLTX

Ever CLAD, N (\%)

Graft loss, N (\%)

\begin{tabular}{|cc} 
Azithromycin (32) & Placebo (37) \\
\hline $51 \pm 3$ & $50 \pm 2$ \\
& \\
$14(44 \%)$ & $22(59 \%)$ \\
$6(19 \%)$ & $8(22 \%)$ \\
$9(28 \%)$ & $6(16 \%)$ \\
$2(6 \%)$ & $0(0 \%)$ \\
$1(3 \%)$ & $1(3 \%)$ \\
& \\
$2(6 \%)$ & $0(0 \%)$ \\
$10(31 \%)$ & $7(19 \%)$ \\
$20(63 \%)$ & $30(81 \%)$ \\
$17(53 \%)$ & $24(65 \%)$ \\
$12(38 \%)$ & $18(49 \%)$
\end{tabular}




\section{FIGURE LEGENDS}

Figure 1: Patient recruitment and samples included in this retrospective analysis of a randomized placebo-controlled trial of azithromycin for the prevention of chronic rejection after lung transplantation

Figure 2: Comparison of the microbiota in the azithromycin and placebo cohorts showing: $(A)$ The relative abundance of bacteria (present $>1 \%$ mean RA) in azithromycin and placebo patients at discharge, $1 \mathrm{Y}$ and 2y. (B) Significant difference in Pseudomonas relative abundance $2 Y$ post-LTx between azithromycin and placebo-treated patients, alongside similar results between cohorts for community diversity and bacterial density.

Figure 3: Evolution of lung microbiota in serial samples from discharge to 1 and 2 years following transplantation independent of azithromycin use, showing $(A)$ bacteria present in mean $\mathrm{RA}>1 \%$ at discharge, $1 \mathrm{Y}, 2 \mathrm{Y}$ and at development of RAS and BOS phenotypes of CLAD. (B) Comparison of Shannon diversity, richness and bacterial density at discharge, $1 \mathrm{Y}, 2 \mathrm{Y}$ and with diagnosis of CLAD.

Figure 4: Association between acute rejection and bacterial dysbiosis. (A) The Shannon diversity, evenness and richness is decreased in patients with biopsy-proven acute cellular rejection. (B) No clear changes in microbiota composition except for the loss of some lowabundance genera.

Figure 5: Univariate correlation analysis between microbial presence and clinically important variables. This indicates associations between airway inflammation and a wide variety of 
genera, but also with Shannon diversity index and Shannon evenness. Red indicates a positive association and blue a negative association. 